\title{
The influence of wet feed distribution on the density, growth rate and growth variability of Tenebrio molitor
}

\author{
D. Deruytter", C.L. Coudron and J. Claeys \\ Department of aquaculture and insect rearing, Inagro, Ieperseweg 87, 8800 Rumbeke-Beitem, Belgium; \\ david.deruytter@inagro.be
}

Received: 4 June 2020 / Accepted: 1 September 2020

(c) 2020 Wageningen Academic Publishers

OPEN ACCESS C) (1) RESEARCH ARTICLE

\begin{abstract}
Mealworm larvae have the potential to be a future food and feed. One of the difficulties in rearing mealworms efficiently, is their need for both dry and wet feed. Extensive research has been done on dry feed and distributing this feed is easy with the existing technology. However, the wet feed (frequently chopped up vegetables) comes with several challenges. The nature of the wet feed (neither solid nor liquid) complicates automation, making manual feeding still the norm in many farms. This may result in an unequal distribution of the wet feed. The goal of this study was to assess the influence of distance to the wet feed (substituted by agar) on density, growth rate and growth rate variability of mealworm larvae. The entire larval growth stage was assessed from less than $1 \mathrm{mg}$ up to more than $100 \mathrm{mg}$, as differences in mobility were expected for different sized larvae. The results indicate that the distribution of the agar plays a major role in the distribution and growth of the larvae, with less larvae and a decrease in growth rate observed when the agar was more than 5 to $10 \mathrm{~cm}$ away from the larvae. Few mealworms were found more than $15 \mathrm{~cm}$ from the agar, and their growth was inhibited resulting in a biomass up to 150 times lower than near the agar. The effects were mainly observed in larvae weighing less than $30 \mathrm{mg}$, heavier mealworms were mobile enough to crawl to and from the agar. Based on the results, and in order to ensure that all larvae sizes grow equally well and are well distributed in the crate, we strongly advise against distances of $10 \mathrm{~cm}$ or more and we recommend the placement of wet feed within $5 \mathrm{~cm}$ from the larvae.
\end{abstract}

Keywords: yellow mealworm, insect rearing, insect production, wet feed distribution

\section{Introduction}

The insect industry, like any industry, needs to use their resources such as time, money and space efficiently. On a farm-scale, this means optimising the production lay-out, improving stacking height, optimal positioning of the crates, automation, etc. Besides these macro level improvements, advancements can also be made on a smaller scale: the rearing crate itself. For an efficient rearing process, it is important to maximize the insect density and minimise the cycle time while maintaining the health and nutritional quality of the insects. To achieve that, it is important to use a feed that provides an optimal and preferably homogeneous growth of individuals. Likewise, the distribution of the feed within a crate will most likely have an effect on the growth and distribution of the insects. Previous research has indicated that the growth and health of insects can be density-dependent (Barnes and Siva-Jothy, 2000). Furthermore, an uneven distribution of the insects may result in uneven use of feed (Behmer et al., 2003) and accumulation of frass which may also pose problems as volatile fatty acids may be released (Weaver et al., 1990). Therefore, it is likely that differences in larval density within a crate may decrease the performance of the insect farm.

One of the challenges in rearing mealworm larvae is that they need both a dry feed and a wet feed (mainly a source of water, but may contain nutrients) in order to thrive. The dry feed may consist of pure wheat bran and may be supplied all at once or every few weeks during the larval stage. Several studies have already been conducted to assess the optimal nutritional composition for mealworm growth. John et al. 
(1979) describes the amount of amino acids needed in the feed and Fraenkel (1955) made a detailed report on the effect of different carbohydrate sources. Morales Ramos et al. (2013) found an optimal diet of $80 \%$ wheat bran and $20 \%$ supplement consisting of $83 \%$ dry potato, $13 \%$ dry egg white, $2 \%$ soy protein and $2 \%$ peanut oil. Even though mealworm larvae are, partially, able to differentiate among different components of feed (Morales Ramos et al., 2013; Murray, 1960), it can be expected that all larvae have access to all components if the dry feed particle size is small and homogeneity is high.

The use of a wet feed increases the growth rate and maximum weight of the larvae (Urs and Hopkins, 1973) and whoever reared mealworms has undoubtedly observed the crowding effect on and near the wet feed. Potato slices are even suggested as lure for sampling and monitoring adult Tenebrio molitor (Baek et al., 2015). For an optimal growth, wet feed should be present at all times during the growth cycle. Chopped up carrots and other vegetables are frequently used for this purpose. Compared to dry feed, the feeding of wet feed is more challenging. To our knowledge, there is no research on the potential influence of different types of wet feed on mealworm growth. Furthermore, because all vegetables are prone to dehydration and microbial decay it must be provided frequently (e.g. daily), and it is difficult to distribute the vegetable pieces evenly in the crate. As a result, distributing wet feed in a farm is difficult and time consuming and many (small-scale) rearing facilities therefore provide wet feed by hand. This frequently results in an uneven distribution or only centre feeding. Automated feeding systems will provide a constant feeding regime in time and space, but it may not be possible to achieve a homogeneous distribution throughout the rearing crate. It may not even be desirable, as this increases the handling time per crate and/or the initial investment cost of automation. For example, if a piston or plunger pump is used to provide the wet feed either the time increases as the outlet has to move across the crate and/or the investment increases as multiple outlets would need to be fitted. To the best of our knowledge, there is no quantitative data on the effect of spatial distribution of wet feed on mealworm density and growth. It can be expected that unevenly spread wet feed will result in either heterogeneous distribution of the mealworms with a higher density near the wet feed or growth retardation of mealworms that are not mobile enough to reach the wet feed (Urs and Hopkins, 1973).

The goal of this study was to assess the influence of distance to the wet feed, substituted by agar, on the density, growth rate and growth rate variability and to quantify the distance at which adverse effects start to occur. A difference in mobility between small and large mealworms was expected, therefore the entire larval growth stage was assessed, starting two weeks after oviposition until pupation. Finally, a comparison was made between mealworms that were fed in a single spot their entire larval stage or only for one week. The former represents a worst-case scenario and indicates what happens when there would be centre feeding during the whole larval phase. The latter provides a better indication of the sensitivity of mealworms of a given size to a single spot feeding.

\section{Materials and methods}

\section{Colony information}

The mealworms used in this study have been bred at the Inagro Insect Research Centre (Rumbeke-Beitem, Belgium) since 2013. They are kept in $60 \times 40 \mathrm{~cm}$ plastic crates (inner surface area of $2,000 \mathrm{~cm}^{2}$ ) at a temperature of $27 \pm 1{ }^{\circ} \mathrm{C}$, $60 \pm 3 \%$ relative humidity and in the dark except during feeding. The animals are fed ad libitum with INSECTUS Mealworm Grow (Mijten nv, Bekkevoort, Belgium) and chopped chicory roots. The $\mathrm{CO}_{2}$ concentration was monitored and kept below 1,500 ppm. In order to gather enough larvae for the experiments, $16 \times 200$ grams of beetles were allowed to lay eggs for $24 \mathrm{~h}$ for 4 consecutive days. After oviposition the eggs and substrate were collected and left at $27{ }^{\circ} \mathrm{C}$ and $60 \%$ relative humidity. During that period no wet feed was provided. After 14 days $( \pm 1$ week old mealworms) all crates were gently homogenised and three subsamples were taken to estimate the number of offspring.

\section{Experiment 1}

The mealworms were distributed in eight crates with at an estimated abundance of 10,000 mealworms (5 larvae/ $\mathrm{cm}^{2}$ ). Each crate was provided with $2 \mathrm{~kg}$ of evenly distributed dry feed (INSECTUS Mealworm Grow) and agar as wet feed. Agar (25 g/l, Brouwland nv, Beverlo, Belgium) was provided ad libitum in a single strip on one side of the crate (Figure 1) of $36 \times 1 \times 1 \mathrm{~cm}(\mathrm{l} \times \mathrm{w} \times \mathrm{h})$ or $36 \times 2 \times 2 \mathrm{~cm}$ when the $1 \times 1 \mathrm{~cm}$ strip was insufficient as wet feed for $24 \mathrm{~h}$. The availability of agar was checked at least on a daily basis. When needed dry feed was added to the crate to avoid any starvation effects.

Each week one of the crates was sacrificed to assess the density, growth, biomass and growth variability. This was done by dividing the crate in seven zones, established in a previous pilot study: 0-2.5 cm (agar), 2.5-5 cm, 5-10 $\mathrm{cm}, 10-15 \mathrm{~cm}, 15-25 \mathrm{~cm}, 25-40 \mathrm{~cm}$ and $40-56 \mathrm{~cm}$ away from the agar strip (Figure 1). The content (substrate + larvae) of each zone was extracted, gently homogenised and a random subsample was taken to determine the total number, average weight and individual weight of the larvae (on average 65 larvae/zone, total 12,904). Two techniques were necessary to determine the individual weight. Mealworms weighing less than $20 \mathrm{mg}$ were photographed using a Dinolite edge 3.0 (AM73915ZMTL; Dino-Lite Europe, Almere, the Netherlands) and the length and width were measured using the DinoCapture 2.0. This 


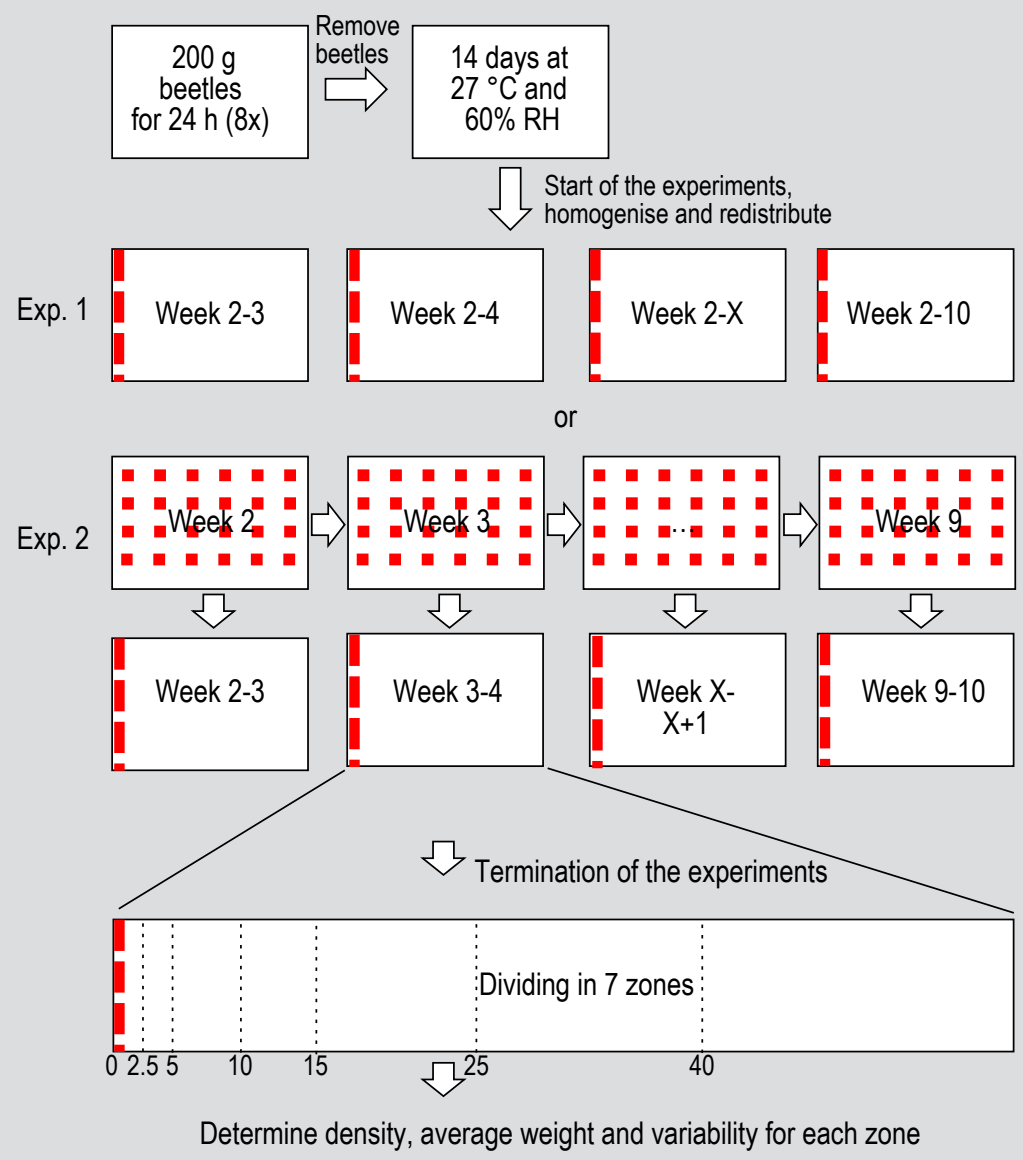

Figure 1. Schematic overview of experiment 1 and 2. For clarity, the overview is only for one replicate while the experiments were replicated 4 times. Red indicates the location of the wet feed ( $25 \mathrm{~g} / \mathrm{l}$ agar).

data was used to estimate individual weight (Supplementary Figure S1). Mealworms weighing more than $20 \mathrm{mg}$ were weighed directly on a Mettler Toledo ME203T (N.V. Mettler-Toledo S.A., Zaventem, Belgium). The number of individual measurements was, on average, 41 per zone. The experiment was terminated when all 8 crates were sacrificed, 8 weeks after the start of the experiment or 10 weeks after the eggs were laid. Data from the last experimental week was not used as there were to many pupae present. The experiment was replicated 4 times on 4 consecutive days (Tuesday to Friday) resulting in a total of 32 crates. This was necessary as not enough larvae were available on a single day.

\section{Experiment 2}

Experiment 2 was similar to experiment 1, but larvae of different sizes were only exposed to the experimental conditions for one week. During the weeks foregoing the experiment, rearing conditions were such that uniformity of the mealworms at the start of the experiment was maximised. The mealworms were fed ad libitum with dry feed and evenly distributed agar and each crate was gently homogenised every two days. Every week mealworms were taken from these homogenous parent batches and placed in a new crate identical as in experiment 1 . In order to avoid the need to add dry feed during the experiment and reduce any potential effects of depletion of the dry feed in some zone, the number of larvae in the experimental crates was decreased with increasing mealworm size from 15,000 to 5,000 (7.5 to 2.5 larvae $\left./ \mathrm{cm}^{2}\right)$. After one week the crate was analysed as in experiment 1 . This was done for 8 weeks in a row to assess different mealworm sizes. Furthermore, the experiment was replicated 4 times on 4 consecutive days (Tuesday to Friday) resulting in a total of 32 crates that were assessed. A schematic representation of both experiments can be seen in Figure 1.

\section{Statistical analysis}

The statistical analyses were performed using R2.12.1 statistical software (https://www.r-project.org/). If necessary, the data were transformed to achieve normal distribution of the residuals and to avoid heteroscedasticity. 
The independent variables were distance to the agar in $\mathrm{cm}$ taken as the distance from the agar to the centre of each sampling zone (e.g. zone $10-15 \mathrm{~cm}=12.5 \mathrm{~cm}$ ) and larval weight at the start of the week. Using weight instead of age ensures a better comparability with other research or industry data as growth curves between different institutes can differ depending on the local conditions. A comparison can be made between size and age in the fitted growth curves. For experiment 1, the total average weight across all sections and replicates of the previous week was taken as the weight at the start of the week. For experiment 2, the average weight of the mealworms was directly calculated via a subsample of the mealworms that were added to the experimental crates from the parent crates. The assessed combinations between the initial weight and the distance to the agar can be found in the supporting information (Supplementary Figure S2).

To improve comparability, the following data transformations were performed. To eliminate the influence of overall number of mealworms in the crate and the difference in width between the sections, density was calculated as the percentage mealworms per centimetre: $100 \times \#$ mealworms in the zone/(\# mealworms in crate $\times$ length of the zone). Growth rate was calculated as the average weight after 7 days divided by the initial weight of the mealworms. Furthermore, the growth rate was divided by the growth rate in the first section $(0-2.5 \mathrm{~cm})$ and multiplied by 100 . This ensured that the growth itself was not modelled but only differences in growth rate in \%, compared to the first zone. Biomass was calculated as the average weight multiplied by the density. This was transformed to $\%$ biomass $/ \mathrm{cm}$ (similar to \% mealworms/cm) via: $100 \times$ biomass in the zone/(total biomass in the crate $\times$ length of the zone). Finally, the coefficient of variation was used as a measure of the variability in individual weight.

The data was analysed using generalised additive modelling (GAM). This technique does not assume a linear relationship between the predictor and response variables and is thus able to model complex relationships. This was done for both experiments and for the density, growth rate, biomass and size variability. A gaussian distribution was used with an identity link function (Equation 1).

$\mathrm{E}[\mathrm{Y} \mid$ initial weight, distance $]=$ intercept $+\mathrm{f}($ initial weight, distance)

With $\mathrm{E}[\mathrm{Y} \mid$ initial weight, distance] the expected value at a given combination of initial weight and distance to agar. The smoothing function $\mathrm{f}$ denotes the combined effect of initial weight and distance to agar. This full model was reduced via backward selection to find the model with the lowest Akaike information criterion (AIC). The latter measures the goodness of fit and model complexity and can include non-significant terms.
Although the GAM models provide an overview of the effect, it does not allow the construction of a mathematical formula that can be used practically in an industrial setting. To ensure that the results of these experiments can be used by other institutes and the industry, the GAM models were used to calculate isolines for the density, biomass and growth. In this way, a tool is provided to calculate at what distance from the agar adverse effects occur. For density and biomass an iso-curve was constructed representing the distance to the agar where the mealworm density or biomass is equal to what would be expected when they are evenly spread throughout the crate $(1.8 \%$ /cm or $100 / 56$, the inner length of the crate). For the growth the $80 \%$ isoline was constructed indicating the distance to the agar where mealworms grew only at $80 \%$ of the rate compared to the larvae at the agar. Finally, to assess specific significant differences between zones at a certain time, an ANOVA with a post-hoc Tukey test was used.

\section{Results}

The growth curves (Figure 2) for the 0 to $2.5 \mathrm{~cm}$ zone (the assumed optimal zone) could best be fitted by a 3-parameter log-logistic model: maximum weight/(1 +

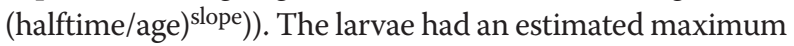
average weight $( \pm \mathrm{SE})$ of $134 \pm 5.5 \mathrm{mg}$ for experiment 1 . The maximum larval weight of experiment 2 was significantly higher than in experiment $1(P<0.001,170 \pm 4.7 \mathrm{mg})$. The time to reach half of the maximum weight and the slope were not significantly different between both experiments (halftime: $6.9 \pm 0.1$ and $7.0 \pm 0.1$ weeks, $P=0.57$; slope $8.1 \pm 0.7$ and $7.2 \pm 0.5, P=0.23)$.

The GAM models were able to fit the density, growth rate and biomass data for both experiments, and explain between 70 and $94 \%$ of the observed variation (predicted vs observed graphs in Supplementary Figure S4, S9 and S14). The initial weight, the distance to the agar and their interaction were always included in the optimal model as this was the model with the lowest AIC (Supplementary Table S1). For all three variables, the most profound effects were observed in mealworms weighing less than $30 \mathrm{mg}$ $\left( \pm 1.5\right.$ on a $\log _{10}$-scale).

The density distribution range was similar between both experiments with $90 \%$ of the observations between 0.36 and $10.88 \%$ in experiment 1 and 0.52 and $7.40 \%$ in experiment 2 with $1.8 \%$ considered a homogeneous density distribution (Figure 3). In both experiments, the highest density was observed closest to the agar, in the first two sections (0-5 $\mathrm{cm}$ ), while the lowest density was observed in the half of the crate furthest away from the agar (Figure 3). This can also be seen on the iso-density lines where the distance between overpopulated and depopulated is between 5 and $17 \mathrm{~cm}$ from the agar depending on the initial weight and experiment (Table 1, Figure 3). Tukey's pair-wise 


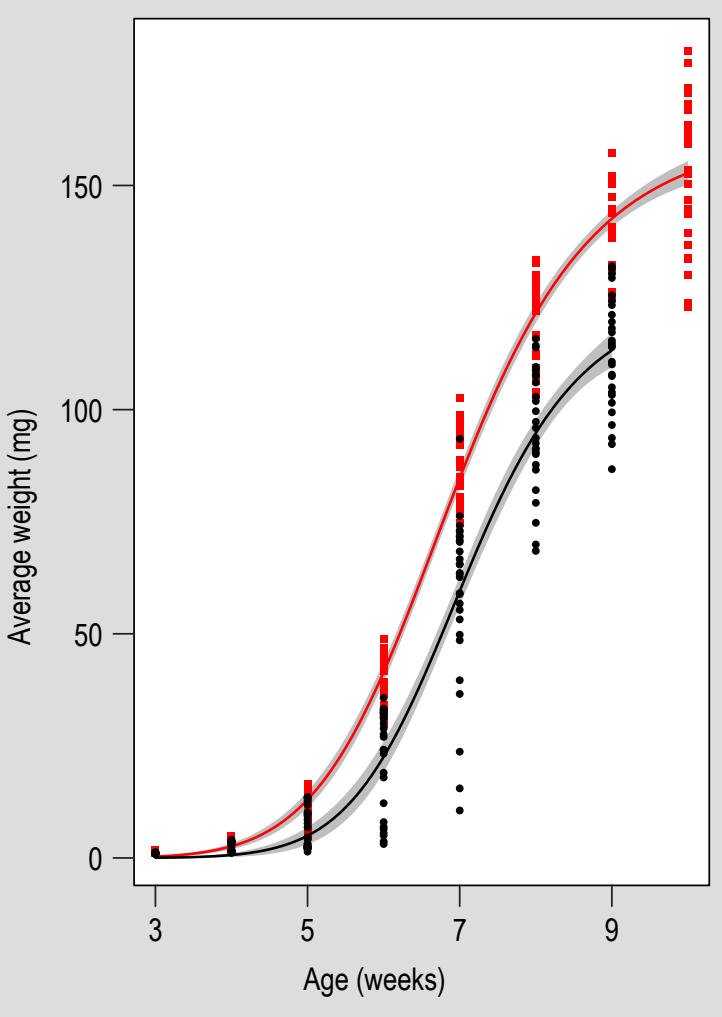

Figure 2. Estimated growth curve based on a 3-parameter loglogistic model. Black $=$ observation and model for experiment 1 (equation: $134 /\left(1+(6.9 / \text { age })^{8.1}\right)$; Red = observation and model for experiment 2 (equation: $170 /\left(1+(7.0 / \text { age })^{7.2}\right)$. Gray area is the $95 \%$ confidence interval.

comparisons revealed significant negative effects as early as the $5-10 \mathrm{~cm}$ zone compared to the $0-2.5 \mathrm{~cm}$ zone $(P<0.05$, Supplementary Figure S6 and S8). When combining the data for all the mealworms with an average initial weight below $30 \mathrm{mg}$, half the mealworms were found within the first $10 \mathrm{~cm}$ of the agar (experiment 1: 50.5 $\pm 5.1 \%$; experiment 2: $52.3 \pm 6.5 \%)$.
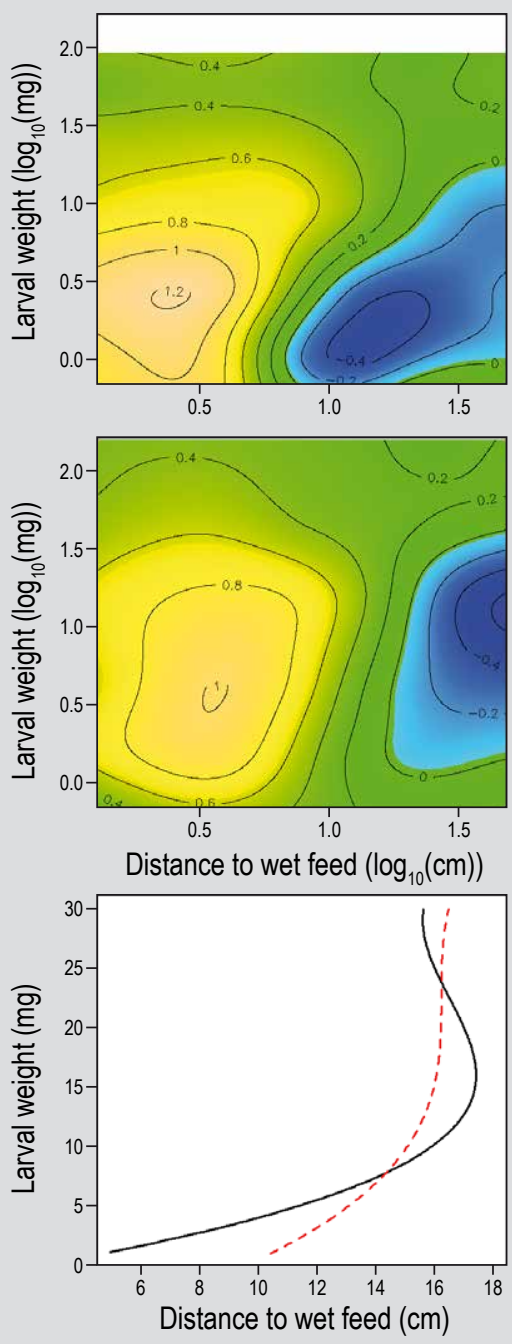

Figure 3. The density model predictions for experiment 1 (top) and 2 (middle) on a $\log _{10}(\%$ mealworms $/ \mathrm{cm}$ ) scale between -0.6 (dark blue) and 1 (orange/white), and iso-density lines for both experiments (bottom): black $=$ experiment 1, red $=$ experiment 2 .

Table 1. Equations to calculate the distribution, $80 \%$ growth or biomass iso-lines up to a larval weight of $30 \mathrm{mg}$ with the distance in centimetres and the weight (W) in milligram. The $80 \%$ iso-growth line of experiment 1 is not presented as a quintic polynomial model was needed.

$\begin{array}{lllllll}\text { Equation } & \text { Variable } & \text { Experiment } & \text { Intercept } & \mathbf{W} & \mathbf{W}^{2} & \mathbf{W}^{\mathbf{3}} \\ 2 & & & & & -0.112 & 1.65 \times 10^{-3} \\ 3 & \text { density } & 1 & 2.52 & 2.29 & -0.0403 & 5.99 \times 10^{-4} \\ 4 & \text { density } & 2 & 9.53 & 0.903 & & \\ 5 & \text { growth } & 2 & 3.06 & 2.29 & -0.0936 & 1.38 \times 10^{-3} \\ 6 & \text { biomass } & 1 & 2.24 & 2.20 & -8.44 \times 10^{-3} & \end{array}$


Similar to the density the highest growth rate was observed at or near the agar. The $80 \%$ iso-growth lines indicate a decrease in growth already at $5 \mathrm{~cm}$ (Table 1, Figure 4), according to Tukey's pair-wise comparison significant declines can start at the 10-15 cm zone compared to the 0-2.5 cm zone $(P<0.05$; Supplementary Figure S11 and S13). The effect on the larval weight was more pronounced for experiment 1 as even negative growth was observed while the minimum in experiment 2 was still a positive growth of $30 \%$.

The biomass distribution range was similar between both experiments with $90 \%$ of the observations between 0.11 and $14.88 \%$ in experiment 1 and 0.08 and $13.09 \%$ in experiment 2 . In both experiments they were broader than
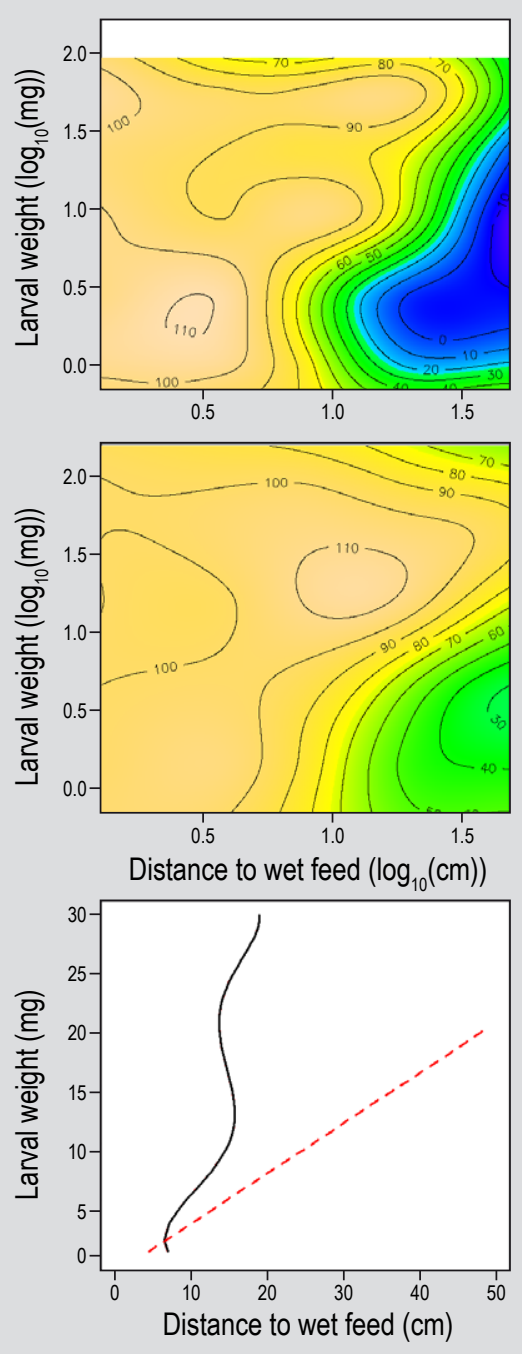

Figure 4. The growth model predictions for experiment 1 (top) and 2 (middle), where the unit for growth is \% growth compared to the control (zone $0-2.5$ ) on a scale between - $10 \%$ (dark blue) and $110 \%$ (orange/white) and the $80 \%$ growth lines for both experiments (bottom): black = experiment 1, red $=$ experiment 2. the ranges for the density distribution. The highest biomass was observed close to the agar with the iso-biomass line between 5 and $20 \mathrm{~cm}(P<0.05$; Figure 5$)$. Tukey's pair-wise comparisons revealed significant negative effects as early as the $5-10 \mathrm{~cm}$ zone compared to the $0-2.5 \mathrm{~cm}$ zone $(P<0.05$; Supplementary Figure S16 and S18).

In the supporting information enlarged and normalised graphs as well as the individual boxplots for each week can be found (Supplementary Figure S5-S8, S10-S13 and S15-S18).

The GAM model could only explain $66 \%$ of the growth variability for experiment 1 and $76 \%$ for experiment 2 . And the optimal model included weight of the larvae,
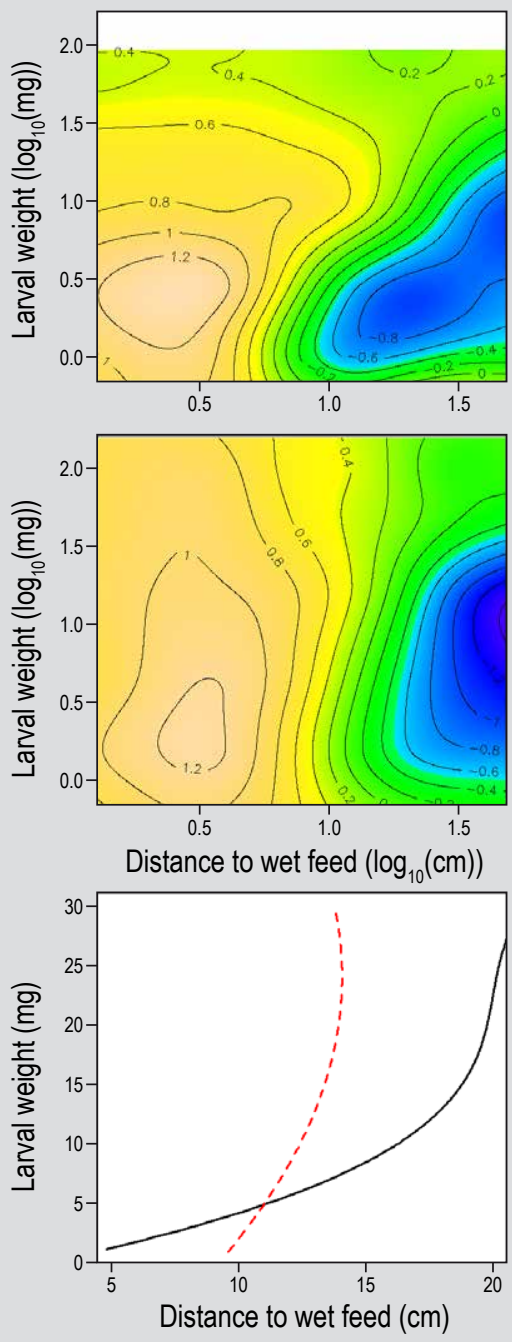

Figure 5. The biomass model predictions for experiment 1 (top) and 2 (middle), on a $\log _{10}(\%$ biomass $/ \mathrm{cm})$ scale between -1.2 (dark blue) and 1.2 (orange/white) and the iso-biomass lines for both experiments (bottom): black = experiment 1 , red = experiment 2. 
distance to the agar and the interaction. For experiment 1 , the coefficient of variation increased with initial weight and with distance to agar with a maximum exceeding $100 \%$ (Figure 6). This was more pronounced for mealworms with an initial weight of more than $10 \mathrm{mg}$. In experiment two a peak in variability was observed in mealworms with a weight between 3 and $30 \mathrm{mg}$ but the influence of distance to agar was moderate compared to experiment 1 , with a maximum of $60 \%$. Overall the coefficient of variation in experiment 1 (54\%) was higher than experiment 2 (31\%). It was observed that the individual measurements in some zones furthest away from the agar in experiment 1 exhibited a dichotomous distribution and therefore may skew the result (Supplementary Figure S3).

\section{Discussion}

T. molitor is a drought resistant insect that can survive by feeding solely on dry feeds such as wheat bran through the use of metabolic water (Hansen et al., 2004, 2006; Murray, 1968). However, when a wet feed source is present the larvae grow faster and with a better feed to larvae conversion rate (Urs and Hopkins, 1973). Many studies currently use carrot slices or another type of vegetable (Deruytter et al., 2019; Houbraken et al., 2016; Osimani et al., 2018). However, vegetables are prone to drying, moulding and have differences in dry matter content, nutrients, etc. As

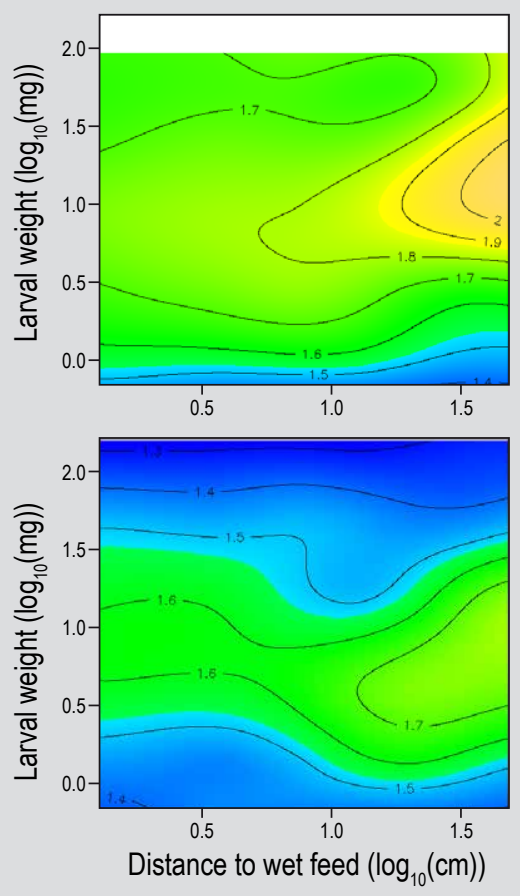

Figure 6 . The growth variability model predictions for experiment 1 (top) and 2 (bottom), where the unit for variability is $\log _{10}$ (coefficient of variation) on a scale between 1.3 and 2 (orange/white). a result, it is nearly impossible to take into account the nutrients obtained via this type of wet feed. The present study is, to our knowledge, the first study using agar as wet feed source. At 25 grams agar per litre water it barely leaks into the dry feed, it is a homogeneous product that can easily be cut into standardised pieces and provides nearly no nutrients. Moreover, it retains most moisture for more than one day although, over time, drying and moulding cannot be prevented completely. The use of agar results in a good overall growth, similar to the best growth in Van Broekhoven et al. (2015). Therefore we suggest the use of agar as a standard wet feed for future mealworm experiments to improve the comparability between experiments.

Previous studies have emphasised the importance of wet feed on the growth of mealworms, the present study emphasises the importance of the distribution of the wet feed in the crate during an experiment and in an industrial setting. As expected, optimal growth performance was observed at, or close to, the wet feed. These areas frequently coincide with a high mealworm density, with more than 5.6 times more larvae than expected based on a homogeneous distribution. As the larvae crowd near the wet feed, there were 3 to 4 times less mealworms than expected in areas further away from the wet feed. The growth rate was also reduced in these low density areas with a reduction starting as early as 5 to $10 \mathrm{~cm}$ away from the wet feed. A negative growth was observed in experiment 1 , this was probably not due to a decrease in the weight of individual mealworms, but more likely due to the larger mealworms migrating towards the wet feed while the smaller, less mobile, mealworms stayed behind.

Due to the apparent correlation between density and growth, the differences in biomass close or far away from the agar are even more profound, up to a factor 150 . With 8 times more biomass than expected close the wet feed (2.5-5 cm zone) and area's with 18 times less biomass than expected further away from the wet feed. The latter are virtual dead zones that may take up a substantial amount of space (frequently more than half of the crate) but will contribute very little to the overall production of mealworms in an industrial setting. Besides the fact that part of the crate is not optimally used, this uneven distribution also results in an uneven consumption of the dry feed which may reduce the growth rate due to a depletion or deterioration of the feed and accumulation of the frass. Although a good growth rate was observed in high biomass areas, Morales-Ramos and Rojas (2015) observed a decreased growth rate and feed efficiency at higher densities. In the current study, the presence of wet feed clearly outweighs the potential negative crowding effects on the mealworm growth in the current study, it may indicate that the maximum growth rate observed in the current study could be improved when the wet feed was spread evenly. Finally, the differences in 
biomass distribution could also influence the local substrate temperature and could cause other indirect crowding effects such as delayed pupation (Tschinkel and Willson, 1971). Further research is needed to assess those effects. It is important to note that this experiment was done at a relative humidity of $60 \%$. At this level, atmospheric absorption of water through the rectum is probably negligible, but our results could differ at a different relative humidity (Hansen et al., 2004; Machin, 1975).

Besides the influence on growth itself, it is clear that feeding in a single spot throughout the entire larval stage (experiment 1 ) resulted in an increase in size variability. In several zones, furthest away from the wet feed, there was a clear dichotomous weight distribution observed near the end of the experiment. This is probably because larger larvae are big and mobile enough to migrate to and from the wet feed and some larvae most likely never reached the wet feed (Supplementary Figure S2). Depending on the end goal of the larvae, an increase in variability may result in additional costs as more efforts would be needed to sieve to the desired size, but also a loss of the smallest mealworms during sieving and it can be expected that the time frame in which the mealworms pupate would be elongated.

In conclusion, the distribution of the wet feed has a major influence on the growth and density distribution of the larvae. Therefore, based on the data in this study, we suggest that larvae, especially those smaller than $10 \mathrm{mg}$, should never be more than $5 \mathrm{~cm}$ away from the nearest wet feed source. This would ensure that all larvae sizes have the possibility to grow equally well and are well distributed throughout the crate. It is strongly advised that a distance of more than $10 \mathrm{~cm}$ away from a feed source is avoided for all larvae sizes. Our results show that well distributed wet feed may increase the optimal or maximum density in a crate, therefore increasing the efficiency of an mealworm farm. However, more research is needed to assess the overall optimal density and how mealworms behave when multiple sources of wet feed are available.

\section{Acknowledgements}

The authors thank L. De Praetere, D. Raniero and M. Cannas. This research was funded by the Interreg $\mathrm{V}$ Flanders - The Netherlands programme co-funded by the European Regional Development Fund: Entomatisation and received funding from the European Union's Horizon 2020 research and innovation programme under grant agreement no. 861976 .

\section{Conflict of interest}

The authors declare no conflict of interest.

\section{Supplementary material}

Supplementary material can be found online at https://doi. org/10.3920/JIFF2020.0049.

Table S1. The Akaike information criterions of the different generalised additive modelling models with single parameters, combined or the interaction.

Figure S1. The average weight of each zone with the average volume of the mealworms in that zone.

Figure S2. The different combinations between distance to wet feed and the initial weight at the start of the experimental week.

Figure S3. The individual weights of one zone in experiment one.

Figure S4. Predicted vs observed graphs for density estimations.

Figure S5. Influence of the initial weight and distance to the wet feed on the density $(\% / \mathrm{cm})$ for experiment 1 .

Figure S6. Visualization of the variability and week by week (W) change in the density of the mealworms in experiment 1 .

Figure S7. Influence of the initial weight and distance to the wet feed on the density $(\% / \mathrm{cm})$ for experiment 2 .

Figure S8. Visualization of the variability and week by week (W) change in the density of the mealworms in experiment 2 .

Figure S9. Predicted vs observed graphs for the growth rate models.

Figure S10. Influence of the initial weight and distance to the wet feed on the growth rate of the mealworms normalized to the growth rate in the first $0-2.5 \mathrm{~cm}$ for experiment 1 .

Figure S11. Visualization of the variability and week by week (W) change in the growth rate of the mealworms in experiment 1 .

Figure S12. Influence of the initial weight and distance to the wet feed on the growth rate of the mealworms normalized to the growth rate in the first $0-2.5 \mathrm{~cm}$ for experiment 2 .

Figure S13. Visualization of the variability and week by week (W) change in the growth rate of the mealworms in experiment 2 . 
Figure S14. Predicted vs observed graphs for the biomass models.

Figure S15. Influence of the initial weight and distance to the wet feed on the biomass distribution $(\% / \mathrm{cm})$ of the mealworms in experiment 1.

Figure S16. Visualization of the variability and week by week (W) change in the biomass of the mealworms in experiment 1 .

Figure S17. Influence of the initial weight and distance to the wet feed on the biomass distribution $(\% / \mathrm{cm})$ of the mealworms in experiment 2 .

Figure S18. Visualization of the variability and week by week (W) change in the biomass of the mealworms in experiment 2 .

\section{References}

Baek, S., Perez, A.E., Turcotte, R.M., White, J.B., Adedipe, F. and Park, Y.L., 2015. Response of Tenebrio molitor (Coleoptera: Tenebrionidae) adults to potato: implications for monitoring and sampling. Journal of Stored Products Research 60: 5-10.

Barnes, A.I. and Siva-Jothy, M.T., 2000. Density-dependent prophylaxis in the mealworm beetle Tenebrio molitor L.(Coleoptera: Tenebrionidae): cuticular melanization is an indicator of investment in immunity. Proceedings of the Royal Society of London. Series B: Biological Sciences 267(1439): 177-182.

Behmer, S.T., Cox, E., Raubenheimer, D. and Simpson, S.J., 2003. Food distance and its effect on nutrient balancing in a mobile insect herbivore. Animal Behaviour 66(4): 665-675.

Deruytter, D., Coudron, C.L. and Teerlinck, S., 2019. Influence of crate size, oviposition time, number of adults and cannibalism on the reproduction of Tenebrio molitor. Journal of Insects as Food and Feed 5(4): 247-255. https://doi.org/10.3920/JIFF2019.0018

Fraenkel, G., 1955. Inhibitory effects of sugars on the growth of the mealworm, Tenebrio molitor $\mathrm{L}$. Journal of cellular and comparative physiology 45: 393-408.

Hansen, L.L., Ramløv, H. and Westh, P., 2004. Metabolic activity and water vapour absorption in the mealworm Tenebrio molitor L.(Coleoptera, Tenebrionidae): real-time measurements by twochannel microcalorimetry. Journal of Experimental Biology 207(3): 545-552.

Hansen, L.L., Westh, P., Wright, J.C. and Ramløv, H., 2006. Metabolic changes associated with active water vapour absorption in the mealworm Tenebrio molitor L.(Coleoptera, Tenebrionidae): A microcalorimetric study. Journal of insect physiology 52(3): 291-299.
Houbraken, M., Sprangherbaes, T., De Clercq, P., Cooreman-Algoed, M., Couchement, T., De Clercq, G., Verbeke, S. and Spanoghe, P., 2016. Pesticide contamination of Tenebrio molitor (Coleoptera: Tenebrionidae) for human consumption. Food chemistry 201: 264-269.

John, A.M., Davis, G.R.F. and Sosulski, F.W., 1979. Protein nutrition of Tenebrio molitor L. XX. Growth response of larvae to graded levels of amino acids. Archives Internationales de Physiologie et de Biochimie 87(5): 997-1004. https://doi.org/10.3109/13813457909070548

Machin, J., 1975. Water balance in Tenebrio molitor, L. larvae; the effect of atmospheric water absorption. Journal of Comparative Physiology 101(2): 121-132.

Morales-Ramos, J.A. and Rojas, M.G., 2015. Effect of larval density on food utilization efficiency of Tenebrio molitor (Coleoptera: Tenebrionidae). Journal of economic entomology 108(5): 2259-2267.

Morales-Ramos, J.A., Rojas, M.G., Shapiro-llan, D.I. and Tedders, W.L., 2013. Use of nutrient self-selection as a diet refining tool in Tenebrio molitor (Coleoptera: Tenebrionidae). Journal of Entomological Science 48(3): 206-221.

Murray, D.R.P., 1960. The stimulus to feeding in larvae of Tenebrio molitor L. Journal of Insect Physiology 4(1): 80-91.

Murray, D.R.P., 1968. The importance of water in the normal growth of larvae of Tenebrio molitor. Entomologia Experimentalis et Applicata 11(2): 149-168.

Osimani, A., Milanović, V., Cardinali, F., Garofalo, C., Clementi, F., Ruschioni, S., Riolo, P., Isidoro, N., Loreto, N., Galarini, R., Moretti, S., Petruzzelli, A., Micci, E., Tonucci, F. and Aquilanti, L., 2018. Distribution of transferable antibiotic resistance genes in laboratory-reared edible mealworms (Tenebrio molitor L.). Frontiers in Microbiology 9: 2702.

Tschinkel, W.R. and Willson, C.D., 1971. Inhibition of pupation due to crowding in some tenebrionid beetles. Journal of Experimental Zoology 176(2): 137-145.

Urs, K.C.D. and Hopkins, T.L., 1973. Effect of moisture on growth rate and development of two strains of Tenebrio molitor L.(Coleoptera, Tenebrionidae). Journal of Stored Products Research 8(4): 291-297.

Van Broekhoven, S., Oonincx, D.G., Van Huis, A. and Van Loon, J.J., 2015. Growth performance and feed conversion efficiency of three edible mealworm species (Coleoptera: Tenebrionidae) on diets composed of organic by-products. Journal of Insect Physiology 73: 1-10.

Weaver, D.K., McFarlane, J.E. and Alli, I., 1990. Repellency of volatile fatty acids present in frass of larval yellow mealworms, Tenebrio molitor L. (Coleoptera: Tenebrionidae), to larval conspecifics. Journal of Chemical Ecology 16(2): 585-593. 
